\title{
Transverse single-spin asymmetries in pion and photon production from proton-proton collisions
}

Daniel PITONYAK*

RIKEN BNL Research Center

E-mail: dpitonyak@quark.phy.bnl . gov

Koichi KANAZAWA

Temple University

E-mail: koichi.kanazawa@temple.edu

\section{Yuji KOIKE}

Niigata University

E-mail: koike@nt.sc.niigata-u.ac.jp

\section{Andreas METZ}

Temple University

E-mail: metza@temple.edu

\begin{abstract}
We analyze the cause of transverse single-spin asymmetries (TSSAs) in processes where a single particle is detected in the final state, especially those in proton-proton $(p p)$ collisions. There are two main approaches used to describe these observables, namely collinear twist-3 (CT3) factorization and Generalized Parton Model (GPM). We argue that within the CT3 framework, TSSAs in high transverse momentum charged and neutral pion production in $p p$ collisions at the Relativistic Heavy Ion Collider (RHIC) are dominated by a fragmentation mechanism. In addition, we demonstrate how TSSAs in direct photon production can allow one to cleanly access the QiuSterman function, test the process dependence of the Sivers function, and distinguish between the CT3 and GPM formalisms.
\end{abstract}

XXIII International Workshop on Deep-Inelastic Scattering 27 April - May 12015

Dallas, Texas

\footnotetext{
* Speaker.
} 


\section{Introduction}

Transverse single-spin asymmetries (TSSAs) (typically denoted $A_{N}$ ) are a spin phenomenon that has been around for close to 40 years, first starting with measurements at Argonne National Lab [1] and FermiLab [2] in the mid-1970s, FermiLab again in the early 1990s [3], and most recently with AGS $[4,5]$ and RHIC $[6,7,8,9,10,11,12,13]$ at Brookhaven National Lab (BNL). The large effects observed with these experiments have proven to be quite a challenge for perturbative QCD (pQCD), which initially predicted extremely small TSSAs within the naïve collinear parton model [14]. However, in the 1980s it was shown that if one went beyond this model and included collinear twist-3 (CT3) quark-gluon-quark correlations in the nucleon, substantial TSSAs could be generated [15]. In the 1990s this CT3 approach was worked out in more detail for protonproton collisions, first for direct photon production [16, 17, 18] and then for pion production [19]. Over the last decade, several other analyses furthered the development of this formalism - see $[20,21,22,23,24,25,26,27,28,29]$ and references therein. During the same time, another mechanism was also put forth to explain TSSAs in proton-proton collisions. This approach involves the Sivers [30], Collins [31], and Boer-Mulders [32] transverse momentum dependent (TMD) functions and became known as the Generalized Parton Model (GPM) - see [33, 34, 35, 36, 37] and references therein. (We mention that, since most likely a rigorous factorization formula involving TMD functions does not hold for single-inclusive processes (which have only one scale), the GPM can only be considered a phenomenological model.) In Sec. 2 we look at the results from these two formalisms, but focus mainly on those from the CT3 framework. In particular, we show how the CT3 fragmentation mechanism can describe the most recent high transverse momentum RHIC data on $A_{N}$ in $p^{\uparrow} p \rightarrow \pi X$ very well both as a function of $x_{F}$ and of $P_{T}$. In addition, we demonstrate that the so-called Qiu-Sterman function dominates $A_{N}$ in $p^{\uparrow} p \rightarrow \gamma X$ and also allows one to test the process dependence of the Sivers function and distinguish between the CT3 and GPM approaches. Finally, we conclude and give an outlook in Sec. 3.

\section{Phenomenological Results for $A_{N}^{\pi}$ and $A_{N}^{\gamma}$}

The causes of TSSAs in $p^{\uparrow} p \rightarrow \pi X$ (denoted $A_{N}^{\pi}$ ) can come from either the incoming protons or the fragmenting pion, but neither of these effects can be disentangled from the other. For many years within the CT3 framework it was assumed that quark-gluon-quark correlations within the transversely polarized proton, embodied by the so-called Qiu-Sterman (QS) function $T_{F}$, were the dominant source of $A_{N}^{\pi}[19,21]$. However, a fit of the QS function to $A_{N}^{\pi}$ data led to a result that was inconsistent with an extraction of a closely related function, the TMD Sivers function $f_{1 T}^{\perp}$, from semi-inclusive deep-inelastic scattering (SIDIS) data, and became known as the "sign mismatch" crisis [38]. An attempt to resolve this issue through more flexible parameterizations of the Sivers function proved unsuccessful [39], and, by looking at $A_{N}$ data on the target TSSA in inclusive DIS [40, 41], it was argued in fact that the QS function could not be the main cause of $A_{N}^{\pi}$ [42]. This led us recently to look at the impact of fragmentation effects from the outgoing pion [43] based on the analytical calculation performed by two of the authors (A.M. and D.P.) [27], and we refer the reader to those two papers for details on the phenomenological and theoretical analyses. The results of the former are shown in Fig. 1 (for $A_{N}^{\pi}$ vs. $x_{F}$ ) and Fig. 2 (for $A_{N}^{\pi}$ vs. $P_{T}$ ). One 

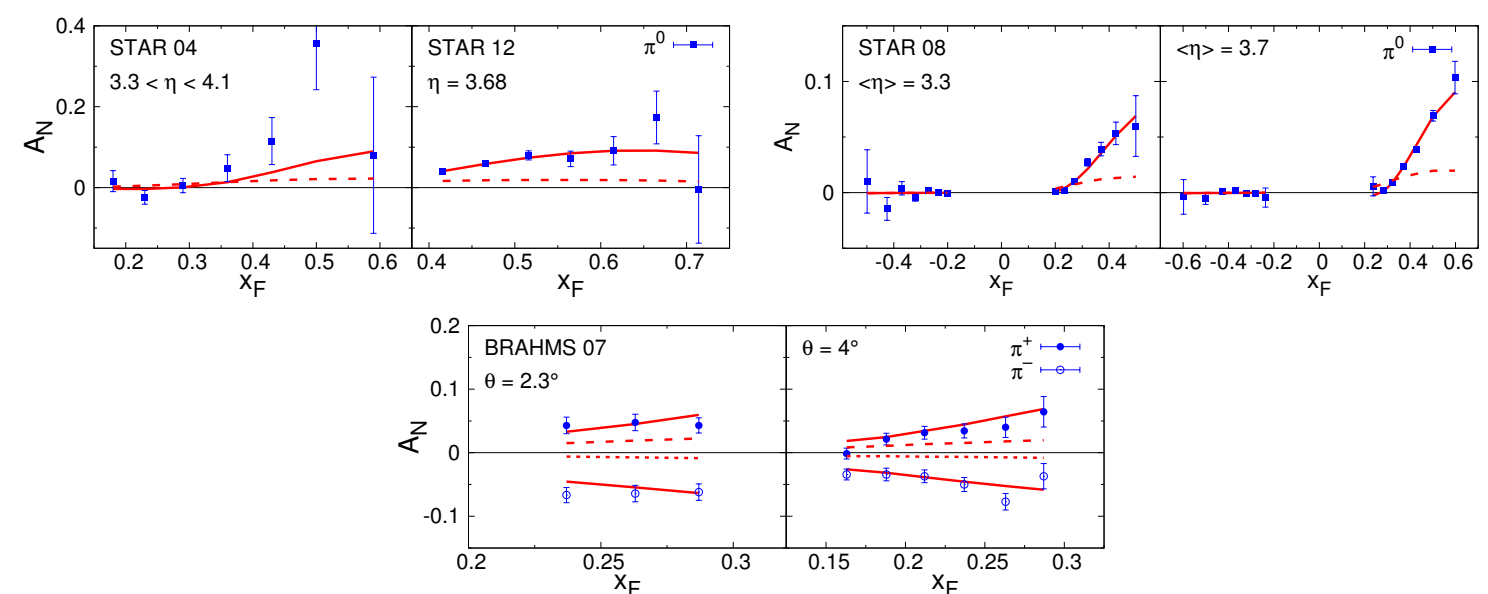

Figure 1: Fit results for $A_{N}^{\pi^{0}}$ (data from [6]) and $A_{N}^{\pi^{ \pm}}$(data from [8]). The dashed line (dotted line in the case of $\pi^{-}$) means $\hat{H}_{F U}^{\mathfrak{S}}$ switched off.

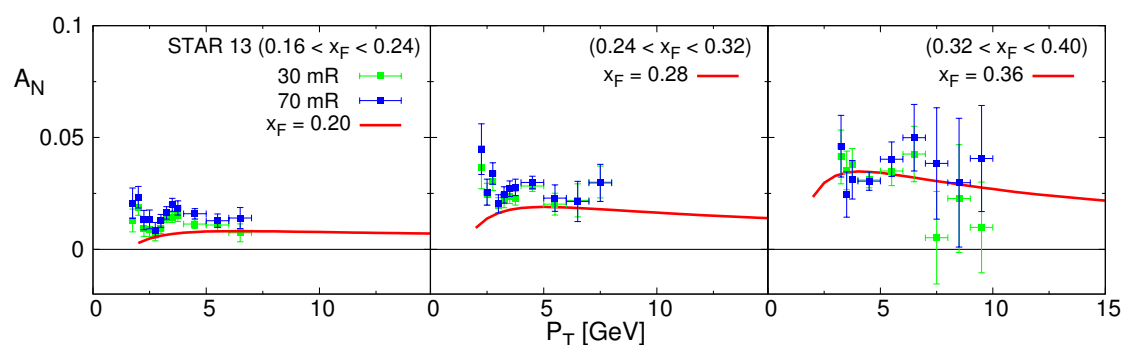

Figure 2: $A_{N}^{\pi^{0}}$ as function of $P_{T}$ at $\sqrt{S}=500 \mathrm{GeV}$ (data from [44]).

sees that we are able to describe all RHIC high transverse momentum $A_{N}^{\pi}$ data very well both as a function of $x_{F}$ and of $P_{T}$. In particular, the dashed curves in Fig. 1 highlight the importance of the quark-gluon-quark fragmentation correlator $\hat{H}_{F U}^{\mathfrak{S}}$, i.e., one does not find agreement with $A_{N}^{\pi}$ if $\hat{H}_{F U}^{\mathfrak{S}}=0$.

The GPM has also analyzed the contribution to $A_{N}^{\pi}$ coming from the transversely polarized proton through the Sivers effect [37] and from the outgoing pion through the Collins effect [36] by using the associated TMD functions extracted from SIDIS and $e^{+} e^{-}$. While neither of these sources alone seem to match all of the RHIC data, one may argue that their sum could give the entire effect. However, keep in mind that in the GPM the Sivers effect has the same sign as the data, while in the CT3 the Sivers-type (QS) piece, due to the sign mismatch, gives a contribution that is opposite to the data (and in fact small in magnitude) if one uses the Sivers function from SIDIS. We will see an immediate consequence of this observation when we now look at TSSAs in $p^{\uparrow} p \rightarrow \gamma X\left(\operatorname{denoted} A_{N}^{\gamma}\right)$.

The direct photon TSSA has been investigated in Refs. [17, 21, 23, 45, 46, 47, 48, 49, 50, 51]. If one ignores fragmentation photons (as should be possible in experiments using isolation cuts), then the only source of $A_{N}^{\gamma}$ comes from the side of the incoming protons. While one of these pieces involves the (soft-gluon pole (SGP) chiral-even) QS function, there are also other contributions, namely (i) soft-fermion pole (SFP) chiral-even quark-gluon-quark functions, (ii) SGP and SFP quark-gluon-quark chiral-odd functions, and (iii) SGP tri-gluon functions. The work in [47] 

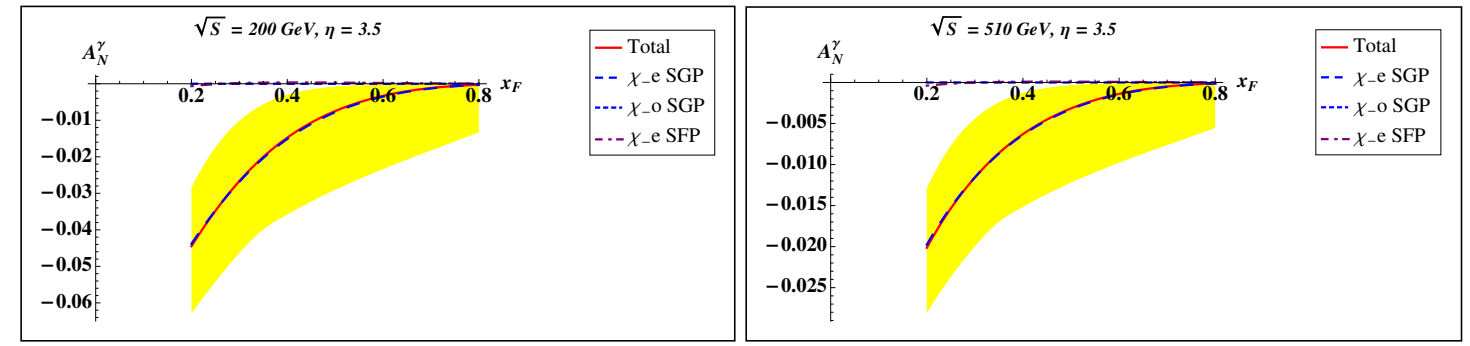

Figure 3: $A_{N}^{\gamma}$ vs. $x_{F}$ at $\sqrt{S}=200 \mathrm{GeV}$ (left) and $\sqrt{S}=510 \mathrm{GeV}$ (right) for fixed $\eta=3.5$. The thick band is our estimate for the error in the asymmetry based on the uncertainty in the Sivers function.

already showed that (iii) is negligible. In [51] we analyzed numerically (i) and (ii) in order to determine what is the dominant source of $A_{N}^{\gamma}$, and we refer the reader to that paper for details on the phenomenology. The results of this study are shown in Fig. 3 for typical RHIC kinematics. One sees that the total asymmetry (solid line) is solely due to the (SGP chiral-even) QS function (dashed line), where we have taken the Sivers function from SIDIS as our input.

Thus, from this observable one should be able to cleanly extract the QS function in order to see if it is consistent with the Sivers effect in SIDIS. Also, since we take the Sivers function from SIDIS as our input for the QS function, a clear negative signal would be a strong indication of the process dependence of the Sivers function [52], which is an important prediction from our current understanding of TMDs. In other words, a clear positive $A_{N}^{\gamma}$ would be equivalent to not seeing a change in sign in the Sivers effect when going from SIDIS to Drell-Yan. In addition, the GPM has also been used to investigate $A_{N}^{\gamma}$, in particular the piece that comes from the Sivers function, and found that the asymmetry is positive and on the order of 5-7\% [37]. Thus, a clear signal from PHENIX and/or STAR, who are currently measuring $A_{N}^{\gamma}[53,54,55]$, would allow one to distinguish between CT3 and GPM.

\section{Summary and Outlook}

Transverse single-spin asymmetries continue to be a spin phenomenon whose cause is still not fully understood. Two main frameworks have been used to analyze $A_{N}$ : collinear twist-3 factorization and Generalized Parton Model. We have demonstrated that the CT3 fragmentation mechanism could be the main source of $A_{N}^{\pi}$. Through this explanation, one is able to resolve the so-called "sign mismatch" between the Qiu-Sterman function and the Sivers function as well as maintain consistency between the spin/azimuthal asymmetries in $p p$ collisions and those in SIDIS and $e^{+} e^{-}$. Within the GPM, $A_{N}^{\pi}$ could be explained as the sum of the Sivers effect and Collins effect. However, the sign of the former agrees with the data, which is opposite to what one finds in the CT3 formalism. An immediate consequence is that the two approaches predict opposite signs for $A_{N}^{\gamma}$. This observable, which PHENIX and STAR are currently measuring, could be a rich source of information, as not only could one distinguish between the CT3 and GPM but also test the process dependence of the Sivers function and cleanly extract the QS function. More experimental and theoretical work must be done in order to understand this truly fundamental observable of TSSAs. For example, one can look at $A_{N}^{\pi}$ in lepton-nucleon collisions at HERMES, JLab, COMPASS, and 
a future Electron-Ion Collider [56, 57, 58, 59] or in high luminosity proton-proton collisions at the proposed AFTER@LHC experiment [60, 61].

\section{Acknowledgments}

This work has been supported by the Grant-in-Aid for Scientific Research from the Japanese Society of Promotion of Science under Contract No. 26287040 (Y.K.), the National Science Foundation under Contract No. PHY-1205942 (K.K. and A.M.), and the RIKEN BNL Research Center (D.P.).

\section{References}

[1] R. D. Klem, J. E. Bowers, H. W. Courant, H. Kagan, M. L. Marshak, E. A. Peterson, K. Ruddick and W. H. Dragoset et al., Phys. Rev. Lett. 36, 929 (1976).

[2] G. Bunce et al., Phys. Rev. Lett. 36, 1113 (1976).

[3] D. L. Adams et al. [E581 and E704 Collaborations], Phys. Lett. B 261, 201 (1991); D. L. Adams et al. [E704 Collaboration], Phys. Lett. B 264, 462 (1991).

[4] K. Krueger et al., Phys. Lett. B 459, 412 (1999).

[5] C. E. Allgower, K. W. Krueger, T. E. Kasprzyk, H. M. Spinka, D. G. Underwood, A. Yokosawa, G. Bunce and H. Huang et al., Phys. Rev. D 65, 092008 (2002).

[6] J. Adams et al. [STAR Collaboration], Phys. Rev. Lett. 92, 171801 (2004) [hep-ex/0310058]; B. I. Abelev et al. [STAR Collaboration], Phys. Rev. Lett. 101, 222001 (2008) [arXiv:0801.2990 [hep-ex]].

[7] S. S. Adler et al. [PHENIX Collaboration], Phys. Rev. Lett. 95, 202001 (2005) [hep-ex/0507073].

[8] J. H. Lee et al. [BRAHMS Collaboration], AIP Conf. Proc. 915, 533 (2007).

[9] I. Arsene et al. [BRAHMS Collaboration], Phys. Rev. Lett. 101, 042001 (2008) [arXiv:0801.1078 [nucl-ex]].

[10] L. Adamczyk et al. [STAR Collaboration], Phys. Rev. D 86, 032006 (2012) [arXiv:1205.2735 [nucl-ex]].

[11] L. Adamczyk et al. [STAR Collaboration], Phys. Rev. D 86, 051101 (2012) [arXiv:1205.6826 [nucl-ex]].

[12] L. C. Bland et al. [AnDY Collaboration], arXiv:1304.1454 [hep-ex].

[13] A. Adare et al. [PHENIX Collaboration], Phys. Rev. D 90, 012006 (2014) [arXiv:1312.1995 [hep-ex]]; Phys. Rev. D 90, 072008 (2014) [arXiv:1406.3541 [hep-ex]].

[14] G. L. Kane, J. Pumplin and W. Repko, Phys. Rev. Lett. 41, 1689 (1978).

[15] A. V. Efremov and O. V. Teryaev, Sov. J. Nucl. Phys. 36, 140 (1982) [Yad. Fiz. 36, 242 (1982)]; Phys. Lett. B 150, 383 (1985).

[16] J.-w. Qiu and G. F. Sterman, Phys. Rev. Lett. 67, 2264 (1991).

[17] J.-w. Qiu and G. F. Sterman, Nucl. Phys. B 378, 52 (1992).

[18] V. M. Korotkiian and O. V. Teryaev, Phys. Rev. D 52, R4775 (1995). 
[19] J.-w. Qiu and G. F. Sterman, Phys. Rev. D 59, 014004 (1999) [hep-ph/9806356].

[20] H. Eguchi, Y. Koike and K. Tanaka, Nucl. Phys. B 752, (2006) [arXiv:hep-ph/0604003].

[21] C. Kouvaris, J. W. Qiu, W. Vogelsang and F. Yuan, Phys. Rev. D 74, 114013 (2006) [arXiv:hep-ph/0609238].

[22] H. Eguchi, Y. Koike and K. Tanaka, Nucl. Phys. B 763, 198 (2007) [arXiv:hep-ph/0610314].

[23] Y. Koike and K. Tanaka, Phys. Lett. B 646, 232 (2007) [Erratum-ibid. B 668, 458 (2008)] [hep-ph/0612117].

[24] Y. Koike and K. Tanaka, Phys. Rev. D 76, 011502 (2007) [hep-ph/0703169].

[25] Y. Koike and T. Tomita, Phys. Lett. B 675, 181 (2009) [arXiv:0903.1923 [hep-ph]].

[26] K. Kanazawa and Y. Koike, Phys. Rev. D 82, 034009 (2010) [arXiv:1005.1468 [hep-ph]]; Phys. Rev. D 83, 114024 (2011) [arXiv:1104.0117 [hep-ph]].

[27] A. Metz and D. Pitonyak, Phys. Lett. B 723, 365 (2013) [arXiv:1212.5037 [hep-ph]].

[28] K. Kanazawa and Y. Koike, Phys. Rev. D 88, 074022 (2013) [arXiv:1309.1215 [hep-ph]].

[29] H. Beppu, K. Kanazawa, Y. Koike and S. Yoshida, Phys. Rev. D 89, 034029 (2014) [arXiv:1312.6862 [hep-ph]].

[30] D. W. Sivers, Phys. Rev. D 41, 83 (1990); Phys. Rev. D 43, 261 (1991).

[31] J. C. Collins, Nucl. Phys. B 396, 161 (1993) [hep-ph/9208213].

[32] D. Boer and P. J. Mulders, Phys. Rev. D 57, 5780 (1998) [hep-ph/9711485]; D. Boer, Phys. Rev. D 60, 014012 (1999) [hep-ph/9902255].

[33] M. Anselmino, M. Boglione and F. Murgia, Phys. Lett. B 362, 164 (1995) [hep-ph/9503290].

[34] M. Anselmino and F. Murgia, Phys. Lett. B 442, 470 (1998) [hep-ph/9808426].

[35] M. Anselmino, M. Boglione, U. D’Alesio, E. Leader, S. Melis and F. Murgia, Phys. Rev. D 73, 014020 (2006) [hep-ph/0509035].

[36] M. Anselmino, M. Boglione, U. D’Alesio, E. Leader, S. Melis, F. Murgia and A. Prokudin, Phys. Rev. D 86, 074032 (2012) [arXiv:1207.6529 [hep-ph]].

[37] M. Anselmino, M. Boglione, U. D’Alesio, S. Melis, F. Murgia and A. Prokudin, Phys. Rev. D 88, 054023 (2013) [arXiv:1304.7691 [hep-ph]].

[38] Z. B. Kang, J. W. Qiu, W. Vogelsang and F. Yuan, Phys. Rev. D 83, 094001 (2011) [arXiv:1103.1591 [hep-ph]].

[39] Z.-B. Kang and A. Prokudin, Phys. Rev. D 85, 074008 (2012) [arXiv:1201.5427 [hep-ph]].

[40] A. Airapetian et al. [HERMES Collaboration], Phys. Lett. B 682, 351 (2010) [arXiv:0907.5369 [hep-ex]].

[41] J. Katich et al. Phys. Rev. Lett. 113, 022502 (2014) [arXiv:1311.0197 [nucl-ex]].

[42] A. Metz, D. Pitonyak, A. Schäfer, M. Schlegel, W. Vogelsang and J. Zhou, Phys. Rev. D 86, 094039 (2012) [arXiv:1209.3138 [hep-ph]].

[43] K. Kanazawa, Y. Koike, A. Metz and D. Pitonyak, Phys. Rev. D 89, 111501(R) (2014) [arXiv:1404.1033 [hep-ph]]. 
[44] S. Heppelmann [STAR Collaboration], PoS DIS 2013, 240 (2013).

[45] X. Ji, J. w. Qiu, W. Vogelsang and F. Yuan, Phys. Rev. D 73, 094017 (2006) [hep-ph/0604023].

[46] K. Kanazawa and Y. Koike, Phys. Lett. B 701, 576 (2011) [arXiv:1105.1036 [hep-ph]].

[47] Y. Koike and S. Yoshida, Phys. Rev. D 85, 034030 (2012) [arXiv:1112.1161 [hep-ph]].

[48] L. Gamberg and Z. B. Kang, Phys. Lett. B 718, 181 (2012) [arXiv:1208.1962 [hep-ph]].

[49] K. Kanazawa and Y. Koike, Phys. Lett. B 720, 161 (2013) [arXiv:1212.3071].

[50] L. Gamberg, Z. B. Kang and A. Prokudin, Phys. Rev. Lett. 110, 232301 (2013) [arXiv:1302.3218 [hep-ph]].

[51] K. Kanazawa, Y. Koike, A. Metz and D. Pitonyak, Phys. Rev. D 91, 014013 (2015) [arXiv:1410.3448 [hep-ph]].

[52] J. C. Collins, Phys. Lett. B 536, 43 (2002) [hep-ph/0204004].

[53] PHENIX Beam Use Proposal: Run-15 and Run-16, May 2014.

[54] RHIC Beam Use Request for Runs 15 and 16: The STAR Collaboration, June 2014.

[55] E. C. Aschenauer, A. Bazilevsky, M. Diehl, J. Drachenberg, K. O. Eyser, R. Fatemi, C. Gagliardi and Z. Kang et al., arXiv:1501.01220 [nucl-ex].

[56] A. Airapetian et al. [HERMES Collaboration], Phys. Lett. B 728, 183 (2014) [arXiv:1310.5070 [hep-ex]].

[57] K. Allada et al. [Jefferson Lab Hall A Collaboration], Phys. Rev. C 89, 042201 (2014) [arXiv:1311.1866 [nucl-ex]].

[58] M. Anselmino, M. Boglione, U. D’Alesio, S. Melis, F. Murgia and A. Prokudin, Phys. Rev. D 89, 114026 (2014) [arXiv:1404.6465 [hep-ph]].

[59] L. Gamberg, Z. B. Kang, A. Metz, D. Pitonyak and A. Prokudin, Phys. Rev. D 90, 074012 (2014) [arXiv:1407.5078 [hep-ph]].

[60] K. Kanazawa, Y. Koike, A. Metz and D. Pitonyak, arXiv:1502.04021 [hep-ph].

[61] M. Anselmino, U. D’Alesio and S. Melis, arXiv:1504.03791 [hep-ph]. 